\title{
Primary signet ring cell carcinoma of the prostate treated by radical cystoprostatectomy and chemoradiotherapy
}

\author{
Sun Wook Kim, MD; Woohyun Kim, MD; Yong-Hyun Cho, MD, PhD; Tae-Jung Kim, MD, PhD; \\ Insuk Woo, MD, PhD; ${ }^{3}$ Dong Wan Sohn, MD, PhD'
}

'Department of Urology, ${ }^{2}$ Department of Hospital Pathology, ${ }^{3}$ Department of Internal Medicine, Yeouido St. Mary's Hospital, College of Medicine, The Catholic University of Korea, Seoul, Republic of Korea

Cite as: Can Urol Assoc J 2016;10(1-2):E204-6. http://dx.doi.org/10.5489/cuai.3122 Published online May 12, 2016.

\section{Abstract}

Primary signet ring cell carcinoma (SRCC) of the prostate is very rare. Although SRCC is primarily found in the stomach and colon, it can also be found in the pancreas, breast, thyroid, bladder, and prostate. We recently diagnosed and treated a case of primary SRCC of the prostate.

A 56-year-old Korean man was referred to our institution for evaluation of a one-month history of hematuria and recently identified bladder mass. Transurethral resection of the bladder tumour was performed and histological and immunohistochemical evaluation revealed a diagnosis of SRCC with tumour invading into the outer half of the deep muscularis propria. After three weeks, the patient had radical cystoprostatectomy with ileal conduit. Tumour involved both prostate and bladder, but the centre of the tumour was located in the prostate. Duodenoscopy and colon fibroscopy both indicated no evidence of tumour origin in the gastrointestinal (GI) tract. Overall, this tumour was regarded as primary SRCC of the prostate. Concurrent chemoradiotherapy (CCRT) using leucovorin and fluorouracil was initiated two months later. The patient eventually developed bone and liver metastases and died of hepatopathy.

\section{Introduction}

Primary signet ring cell carcinoma (SRCC) of the prostate is very rare. It was first reported in 1979 and is estimated to occur in $2.5 \%$ of cases of adenocarcinoma of the prostate. ${ }^{1}$ Although SRCC is primarily found in the stomach and colon, it can also be found in the pancreas, breast, thyroid, bladder, and prostate. $^{2}$ We recently diagnosed and treated a case of primary SRCC of the prostate.

\section{Case report}

In July 2013, a 56-year-old Korean man was referred to our institution for evaluation of a one-month history of hematuria and recently identified bladder mass. The patient had no concomitant medical problems or family history of malignancy. The dynamic abdomen and pelvis computed tomography (CT) scan showed an enlarged prostate $(5.0 \mathrm{x}$ $5.2 \mathrm{~cm}$ ) with irregular, ill-defined margins protruding into the bladder base with calcification. Physical examination, including digital rectal exam, was unremarkable. Serum prostate-specific antigen level was $0.64 \mathrm{ng} / \mathrm{mL}$

Transurethral resection of the bladder tumour was performed. Cystoscopy revealed multiple irregular masses in the prostate and bladder base. Grossly, the epicentre of the tumour was located in the prostate and histological and immunohistochemical evaluation revealed a diagnosis of SRCC with tumour invading into the outer half of the deep muscularis propria.

Two weeks later, duodenoscopy and colonoscopy were performed for evaluation of the Gl tract and no evidence of tumour was found. In addition, there were no metastatic lesions on chest CT scan. Invasion of bilateral seminal vesicles was identified on bladder magnetic resonance imaging (MRI) (Fig. 1). Therefore, we diagnosed SRCC of prostate origin, and radical cystoprostatectomy with ileal conduit was performed.

Pathologically, the tumour cells were comprised of loosely dispersed, neoplastic epithelial cells containing intracytoplasmic mucin that displaced the nuclei towards the periphery. The resulting crescent-shaped nuclei were consistent with SRCC (Fig. 2). There was no involvement of the ureters or the urethra; however, tumour invaded perirectal fat and metastasis was found in one of nine pelvic lymph nodes. The results of immunohistochemical stainings implied the possibility of colorectal origin, since CDX-2 and CK20 were positive, while CK-7, TTF-1, and AMACR were negative (Figs. 3, 4; Table 1). Serum carcinoembryonic anti- 


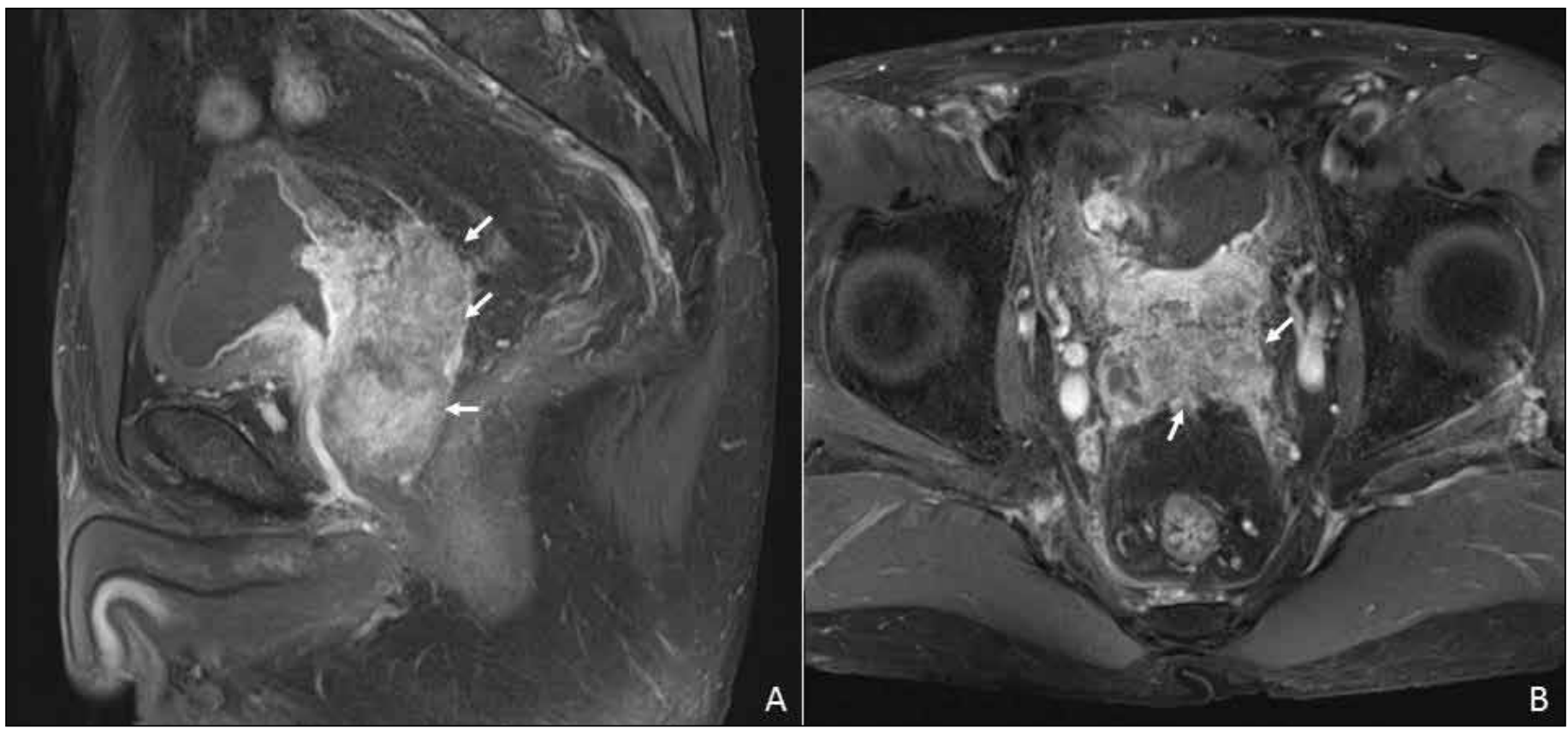

Fig. 1. Enhanced T1WI (A), and T1WI (B) bladder MRI. MRI shows heterogeneous enhancement in basal posterior wall of the urinary bladder. The large mass (arrow) invades bilateral seminal vesicles and fat near the bladder, seminal vesicles, and prostate gland.

gen (CEA) level was normal $(1.56 \mathrm{ng} / \mathrm{ml})$. Tumour involved both prostate and bladder, but the centre of the tumour was located in the prostate.

The patient was referred to oncology for CCRT using leucovorin and fluorouracil. Multiple bone and liver metastases were found 24 months after chemotherapy, and the patient eventually died due to hepatopathy.

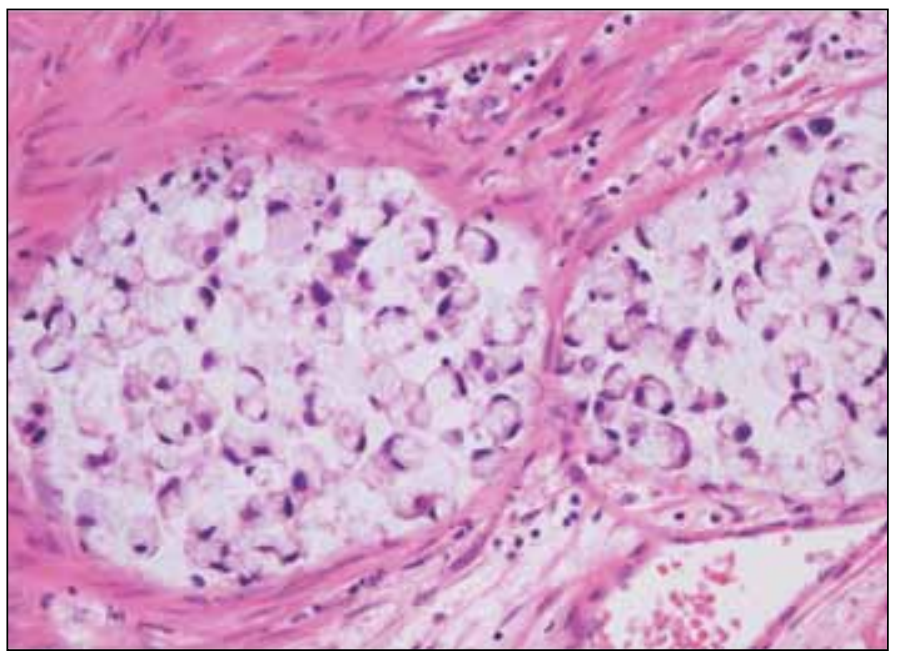

Fig. 2. SRCC of the prostate on Hematoxylin and Eosin staining (x400). Signet ring cells (arrow) have large clear cytoplasmic vacuoles that displace and compress cell nuclei into a crescent shape.

\section{Discussion}

SRCC is a tumour subtype characterized by large intracytoplasmic vacuoles full of mucin that displace nuclei to the periphery of cells. Most SRCCs have been found in the GI tract; primary SRCC is an extremely rare subtype, with about 60 cases previously reported in the literature. ${ }^{3,4}$

Signet ring cells are more frequently seen focally within otherwise high-grade prostate cancers and should be considered as part of the Gleason scoring. ${ }^{5}$ Gleason score sum of 9 or 10 is typical and is usually associated with a high stage, early spread to bones and viscera, and poor outcomes. The largest series to date has shown a three-year survival of $<30 \%$ among 17 cases. $^{6}$

\begin{tabular}{lc}
\hline \multicolumn{1}{l}{ Table 1. Results of immunohistochemical staining } \\
\hline Immunostain & Result \\
\hline Bcl2 & Negative \\
c-erbB2 & Negative (1+) \\
Cyclin D1 & Positive \\
EGFR & Positive (2+) \\
Ki-67 & $60 \%$ \\
P53 & Positive \\
AMACR & Negative \\
CK-7 & Negative \\
CK-20 & Positive \\
CDX-2 & Positive \\
TTF-1 & Negative \\
\hline
\end{tabular}




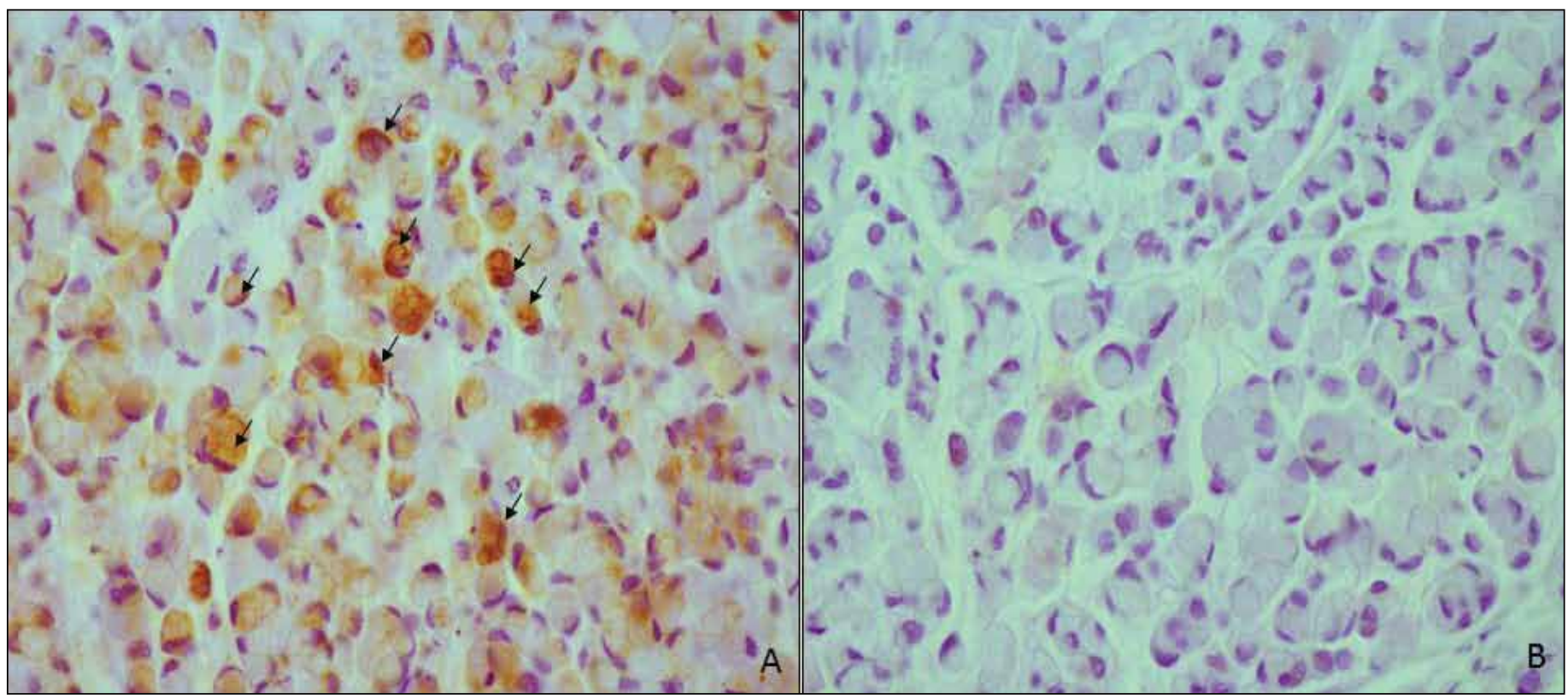

Fig. 3. CK20 positive (A) and CK-7 negative (B) on immunohistochemical staining (x400). Signet ring cells (arrow) stained with CK20 and negative for CK-7.

Clinically, primary SRCC of the prostate presents with the same classic obstructive and irritative symptoms as other common adenocarcinomas. Based on previously reported cases, the mean age of patients is 68.2 years (range: $50-84$ years). Our patient had urinary frequency and nocturia, and was 61 years old at the onset of symptoms.

Because of its rarity, no recommended treatment has been established. Conventional therapy for prostate cancer has generally been used, and prognosis is poor, with mean survival of 29 months. It has been reported that SRCC of the prostate rarely responds to traditional hormonal manipulation, such as androgen blockade. ${ }^{7,8}$ Instead of hormonal therapy, we recommended CCRT due to possible GI tract origin.

Primary SRCCs of the prostate have a poor prognosis even when treated with aggressive therapy. Fujita et al reported that, in primary SRCC of the prostate, the survival rate is about $55 \%$ at three years and decreases to $12 \%$ at five years. ${ }^{9}$

\section{Conclusion}

Primary SRCC of the prostate is an extremely rare subtype and has a poor prognosis even when treated with aggressive therapy. Instead of conventional hormonal therapy, aggressive treatment and close follow-up are necessary for patients with SRCC of the prostate.

Competing interests: The authors declare no competing financial or personal interests.
This paper has been peer-reviewed.

\section{References}

1. Torbenson M, Dhir R, Nangia A, et al. Prostatic carcinoma with signet ring cells: A clinicopathologic and immunohistochemical analysis of 12 cases, with review of the literature. Mod Pathol 1998;11:552-9.

2. Warner JN, Nakamura LY, Pacelli A, et al. Primary signet ring cell carcinoma of the prostate. Mayo Clin Proc 2010;85:1130-6. http://dx.doi.org/10.4065/mcp.2010.0463

3. Berney DM, Montironi R, Egevad L. Pathology in prostate research: optimizing the pathological data. Acta Oncol 2011;50 Suppl 1:49-52. http://dx.doi.org/10.3109/0284186X.2010.525223

4. Humphrey PA. Histological variants of prostatic carcinoma and their significance. Histopathology 2012;60:59-74. http://dx.doi.org/10.1111/i.1365-2559.2011.04039.x

5. Kwon WA, Oh TH, Ahn SH, et al. Primary signet ring cell carcinoma of the prostate. Can Urol Assoc J 2013;7:E768-71. http://dx.doi.org/10.5489/cuai.630

6. Saito S, Iwaki H. Mucin-producing carcinoma of the prostate: Review of 88 cases. Urology 1999;54:141-4. http://dx.doi.org/10.1016/S0090-4295(98)00595-0

7. Lilleby W, Axcrona K, Alfsen GC, et al. Diagnosis and treatment of primary signet ring cell carcinoma of the prostate. Acta Oncol 2007;46:1195-7. http://dx.doi.org/10.1080/02841860701367886

8. Roldán AM, Nú-ez NF, Grande E, et al. A primary signet ring cell carcinoma of the prostate with bone metastasis with impressive response to FOLFOX and cetuximab. Clin Genitourin Cancer 2012;10:199-201. http://dx.doi.org/10.1016/i.dgc.2012.01.006

9. Fuijta K, Sugao H, Gotoh T, et al. Primary signet ring cell carcinoma of the prostate: Report and review of 42 cases. Int J Urol 2004;11:178-81. http://dx.doi.org/10.1111/i.1442-2042.2003.00763.x

Correspondence: Dr. Dong Wan Sohn, Department of Urology, Yeouido St. Mary's Hospital, The Catholic University of Korea, Seoul, Korea; uroking@naver.com 